\title{
Attendere prima di usare l'antibiotico nelle infezioni delle vie respiratorie pediatriche non peggiora la prognosi e ridu- ce il consumo di farmaci non necessari: i risultati di un RCT
}

Mas-Dalmau G, Villanueva López C, Gorrotxategi Gorrotxategi P, et al.

Delayed antibiotic prescription for children with respiratory infections: a randomised trial

Pediatrics. 2021;147(3):e20201323

La prescrizione di antibiotici in età pediatrica è elevata e potrebbe essere ridotta poiché le malattie respiratorie spesso non necessitano di intervento farmacologico. Questo studio pragmatico svolto nelle cure primarie in Spagna ha confrontato tre bracci di intervento: prescrizione antibiotica ritardata, immediata o nessuna prescrizione; il follow-up durato 30 giorni non ha evidenziato differenze nella durata della malattia o nella severità dei sintomi nei tre gruppi di bambini. Nonostante la buona qualità metodologica, l'RCT potrebbe essere inficiato da un non trascurabile bias di selezione; nonostante ciò, diversi altri studi sullargomento conducono a simili risultati.

Waiting before using the antibiotic in pediatric respiratory tract infections does not worsen the prognosis and reduces the consumption of unnecessary drugs: the results of an RCT

The prescription of antibiotics in pediatric age is high and could be reduced as respiratory diseases often do not require pharmacological intervention. This pragmatic study carried out in primary care in Spain compared three intervention arms: delayed antibiotic prescription, immediate or no prescription; the 30-day follow-up revealed no differences in the duration of the disease or in the severity of symptoms in the three groups of children. Despite the good methodological quality, the RCT could be affected by a significant selection bias; nevertheless, several other studies on the subject lead to similar results.

\section{Metodo}

\section{Obiettivo (con tipo studio)}

Studio clinico randomizzato multicentrico volto a valutare l'efficacia della prescrizione antibiotica ritardata (DAP) rispetto alla prescrizione antibiotica immediata (IAP) e alla non prescrizione antibiotica (NAP) nei bambini con infezioni non complicate delle vie respiratorie.

\section{Popolazione}

Pazienti di età compresa tra 2 e 14 anni valutati presso ambulatori di pediatria delle cure primarie per faringite, rinosinusite, bronchite acuta, otite media acuta per i quali il curante avesse il ragionevole dubbio sulla necessità di prescrivere l'antibiotico. I pediatri con disponibilità di test rapido per streptococco potevano includere solo pazienti con rinosinusite, bronchite acuta e otite media acuta. Il reclutamento è stato condotto in 39 centri di primo livello in Spagna.

\section{Esposizione}

I pazienti sono stati randomizzati per ricevere 3 possibili regimi prescrittivi:

Prescrizione antibiotica ritardata (DAP): la terapia antibiotica doveva essere iniziata dai genitori solo se:

1) il bambino non mostrava segni di miglioramento dopo 4, 7 , 15 o 20 giorni dall' esordio dei sintomi rispettivamente di otite media, faringite, rinosinusite o bronchite acuta;

2) il bambino presentava temperatura $\geq 39^{\circ} \mathrm{C}$ per oltre $24 \mathrm{~h} \mathrm{o}$ compresa tra $38-39^{\circ} \mathrm{C}$ per oltre $48 \mathrm{~h}$;

3) il bambino mostrava franchi segni di peggioramento clinico. Prescrizione antibiotica immediata (IAP): la terapia antibiotica veniva iniziata immediatamente.

Nessuna prescrizione antibiotica (NAP): il pediatra non effettuava prescrizione antibiotica. Il tipo di molecola antibiotica utilizzata era appannaggio di ciascun pediatra (senza protocollo uniforme).

\section{Outcome/Esiti}

Obiettivo principale: valutazione della durata dei sintomi e della gravità dell'infezione (da parte dei genitori tramite intervista telefonica) in un arco di 30 giorni.

Obiettivi secondari: uso di antibiotici, soddisfazione e percezione dell'efficacia della terapia prescritta da parte della famiglia, visite aggiuntive da parte del pediatra di base e complicanze correlate all'infezione (diagnosi di polmonite, ascesso, cellulite, accesso al pronto soccorso, ricovero ospedaliero) nei 30 giorni successivi.

\section{Tempo}

Da giugno 2012 a giugno 2016. I pazienti sono stati seguiti con interviste telefoniche al giorno 2 e 30 ; in caso di mancata risoluzione dei sintomi venivano eseguite interviste supplementari ai giorni 7,15 e 22 .

\section{Risultati principali}

Sono stati inclusi nell'analisi 436 bambini con un' età media di 6 anni (51\% femmine); il 51\% aveva una diagnosi di otite media acuta, il 34\% di faringite, il 9\% di bronchite acuta e il $6 \%$ di rinosinusite. La durata media dei sintomi è stata uguale nei 3 gruppi (8.3 giorni nella DAP, 8.3 giorni nella IAP e 7.9 giorni nella NAP) come anche la durata media dei sintomi severi (12.4 nella DAP, 10.1 giorni nella IAP e 10.9 giorni nella NAP) senza differenze statisticamente significative $(\mathrm{p}=0.539)$. Il ricor- 
so all'antibiotico è stato significativamente più alto $(\mathrm{p}=<0.001)$ nel gruppo IAP (96\%) rispetto a DAP e NAP (25\% e $12 \%)$; nel gruppo DAP la durata media tra randomizzazione e assunzione dell'antibiotico è stata di due giorni. Le complicanze, la durata e il tipo di antibiotico prescritto, le visite aggiuntive dal pediatra ed il gradimento del trattamento effettuato sono stati simili nei tre gruppi; l'uso di farmaci sintomatici è stato minore nel gruppo IAP (73\%) rispetto agli altri due gruppi (93\% DAP e $86 \%$ NAP). Gli effetti avversi gastrointestinali sono risultati maggiori nel gruppo IAP.

\section{Conclusioni}

Non è stata riscontrata alcuna differenza statisticamente significativa per quanto riguarda la durata e la severità dei sintomi nei pazienti con infezione respiratoria non complicata che hanno ricevuto DAP rispetto a NAP o IAP. Sicuramente con la DAP si riducono l'utilizzo di terapia antibiotica (e il rischio di resistenze batteriche) e gli effetti avversi gastrointestinali.

\section{Altri studi sull'argomento}

Una revisione sistematica della Cochrane Library del 2017 [1]ha valutato gli effetti di una prescrizione ritardata dell'antibiotico in caso di infezioni del tratto respiratorio rispetto alla prescrizione immediata. Sono stati analizzati 11 studi su un totale di 3.555 pazienti (adulti e pediatrici) curati nell'ambito delle cure primarie e dei reparti di pronto soccorso: il trattamento antibiotico differito ha portato a una riduzione nel numero di antibiotici utilizzati rispetto alla prescrizione immediata (31\% vs 93\%) senza differenze per quanto riguarda il controllo dei sintomi, la comparsa di complicanze cliniche e la soddisfazione del paziente. Solo i sintomi correlati a otite media e faringodinia hanno mostrato un modesto beneficio dal trattamento antibiotico immediato. Gli autori concludono, pertanto, consigliando di adottare una strategia di prescrizione ritardata nei casi dubbi con il fine di ridurre l'utilizzo dell'antibiotico, mantenendo comunque alto il livello di soddisfazione del paziente. Uno studio condotto nell'ambito delle cure primarie in Italia nel 2016 [2] mostra come l'aggiunta della conta dei globuli bianchi (GB) nello studio del medico di famiglia aggiunga molte possibilità di ridurre la prescrizione di antibiotici nelle infezioni delle vie respiratorie anche nei casi in cui si sia già adottato un regime di prescrizione ritardata (dopo 48 ore di febbre). Sono stati arruolati 792 pazienti (età media 6 anni, range 2-14 anni) randomizzati in due gruppi: uno destinato ad assumere l'antibiotico dopo 48 ore di febbre e l'altro destinato a ricevere l'antibiotico solo in caso di una conta di GB $>15.000 / \mathrm{mm} 3$ a 48 ore dall'esordio della febbre oppure in caso di persistenza di febbre elevata e scadimento delle condizioni generali al follow-up. In questo secondo gruppo si è osservata una riduzione della prescrizione antibiotica del $77 \%$ senza differenze nei tassi di risoluzione del quadro clinico o dell'insorgenza di complicanze. Dal 2007 le linee guida (LG) della Regione Emilia Romagna hanno modificato la gestione dell'otite media acuta introducendo la strategia wait-and-see e della faringite acuta e limitando la terapia antibiotica immediata per la faringite acuta alla positività al tampone rapido per lo Streptococco Beta Emolitico di gruppo A (SBEA) o a un punteggio clinico al Mac Isaac score elevato suggestivo per faringite di origine batterica $[3,4]$. L'applicazione di queste LG ha permesso nel tempo di ridurre in modo significativo la prescrizione di antibiotici (-37\% dal 2005 al 2019) senza che questo abbia comportato un aumento delle complicanze (otomastoidite, malattia reumatica). Uno studio condotto nel 2006 negli USA [5] ha arruolato 283 bambini di età 6 mesi-12 anni con diagnosi di otite media acuta posta in Pronto Soccorso, randomizzandoli tra prescrizione antibiotica immediata e strategia wait-and-see (prescrizione con indicazione all' avvio dopo $48 \mathrm{~h}$ in caso di mancato miglioramento, a discrezione del genitore); non è stata osservata differenza in termini di comparsa di febbre, otalgia né di accesso ad ulteriori visite mediche non previste. La terapia antibiotica è stata risparmiata nel $62 \%$ dei casi nel gruppo DAP; nel gruppo IAP il 13\% dei genitori non ha seguito le indicazioni di prescrizione.

\section{Che cosa aggiunge questo studio}

Per le infezioni non complicate delle vie respiratorie una condotta di attesa non peggiora la prognosi e permette di ridurre l'uso degli antibiotici in età pediatrica. La quantificazione del ricorso agli antibiotici è però solo un esito secondario e questo potrebbe inficiare la forza della conclusione.

\section{Commento}

\section{Validità interna}

Disegno dello studio: la qualità metodologica dello studio è buona. Tra i punti di forza: la lista di randomizzazione centralizzata e generata in modo adeguato, stratificata per blocchi e per patologia; il bassissimo numero di persi al follow-up (2.5\% e ben bilanciati tra i tre gruppi), l'utilizzo di schede validate da studi clinici precedenti per valutare la gravità dei sintomi e il grado di soddisfazione dei genitori. L'analisi è stata fatta per intention to treat. Uno dei limiti maggiori dello studio riguarda i criteri di esclusione: in particolare non è specificato sulla base di quali criteri venisse definita l'infezione "complicata" e la prescrizione immediata dell'antibiotico senza inclusione del paziente e randomizzazione. Questo potrebbe aver costituito un importante bias di selezione, tanto più che le infezioni trattate nello studio (per tipologia e gravità) costituiscono l'attività prevalente di un pediatra di famiglia (per lo meno in epoca pre-covid) e ciascun centro avrebbe arruolato soli 11 bambini in quattro anni di studio. Inoltre, l'85\% dei pazienti arruolati era affetto da otite media o faringite, il che può aver inficiato i risultati per quanto riguarda bronchite e rinosinusite (come sottolineano gli autori stessi). Infine, lo studio non era in cieco e i pazienti sono stati seguiti con contatti telefonici fino alla risoluzione dei sintomi, cosa che può aver ridotto l'ansia dei genitori e il ricorso a visite mediche supplementari o aver ridotto il ricorso all'antibiotico anche nel braccio DAP.

Esiti: gli esiti considerati sono ben definiti e clinicamente rilevanti, tuttavia gli esiti primari sono legati alla percezione dei genitori senza nessun parametro clinico oggettivo mentre la quantificazione del ricorso agli antibiotici e delle complicanze sono esiti secondari. Peraltro le complicanze riconosciute tra gli esiti secondari sono eventi rari in patologie a risoluzione tendenzialmente spontanea per cui la numerosità del campione difficilmente avrebbe potuto riscontrare differenze tra i tre bracci.

Conflitto di interesse: gli autori dichiarano di non avere conflitti di interesse. 


\section{Trasferibilità}

Popolazione studiata: sovrapponibile a quella che accede ai nostri ambulatori, come anche per l'elevato consumo medio di antibiotici, tuttavia maggiore in Spagna (31.4 prescrizioni ogni 1.000 abitanti vs 23.4 ogni 1.000 abitanti in Italia vs 21.7 ogni 1.000 abitanti in Europa); in questo Paese la pratica della prescrizione antibiotica ritardata non è comune. In Italia il $41.4 \%$ della popolazione pediatrica riceve almeno una prescrizione di terapia antibiotica all'anno (dati AIFA 2017). Il grande pregio dello studio è che viene condotto proprio in ambito territoriale dove, più che in ambito ospedaliero, si può puntare a una riduzione delle prescrizioni antibiotiche non necessarie (per verosimile minor gravità delle infezioni trattate, per la possibilità di valutare ripetutamente il caso da parte dello stesso medico) e dove è spesso difficile disegnare studi clinici dal solido impianto.

Tipo di intervento: l'intervento descritto è già attuato in Italia, anche se con difformità regionali; in particolare l'Emilia Romagna ha attuato già da più di 15 anni percorsi di sorveglianza dell'appropriatezza prescrittiva (in particolare approccio di vigile attesa nelle otiti, utilizzo di tamponi rapidi nell'ambulatorio del pediatra per lo SBEA e riduzione della durata della terapia antibiotica nelle otiti medie e faringotonsilliti) che hanno permesso di ridurre nel tempo le prescrizioni antibiotiche per le infezioni delle alte vie respiratorie [6].

1. Spurling GK, Del Mar CB, Dooley L, et al. Delayed antibiotic prescriptions for respiratory infections. Review Cochrane Database Syst Rev 2017;9:CD004417. https://doi.org/10.1002/14651858.CD004417. pub5

2. Cioffi L, Limauro R, Sassi R, et al. Decreased antibiotic prescription in an Italian pediatric population with nonspecific and persistent upper respiratory tract infections by use of a point-of-care white blood cell count in addition to antibiotic delayed prescription strategy. Glob Pediatr Health 2016;3:2333794X15771

3. Di Mario S, Gagliotti C, Moro ML, a nome del Gruppo "ProBA". La faringotonsillite in età pediatrica; Aggiornamento delle linee guida della Regione Emilia-Romagna. Medico e Bambino 2015;34(7):442-447

4. Di Mario S, Gagliotti C, Moro ML, a nome del Gruppo "ProBA". Otite media acuta in età pediatrica; Aggiornamento della Linea Guida della Regione Emilia-Romagna . Medico e Bambino 2016;35(1):35-40

5. Spiro DM, Tay KY, Arnold DH, et al. Wait and see prescription for the treatment of acute otitis media. A randomized controlled trial. JAMA 2006;296:1235-1241

6. Di Mario S, Gagliotti C, Buttazzi R, et al. Reducing antibiotic prescriptions in children is not associated with higher rate of complications. Eur J Pediatr 2021;180:1185-1192

Scheda redatta dal gruppo di lettura di Milano:

Riccardo Cazzaniga, Valeria D’Apolito, Valentina Decimi, Gian Piero Del Bono, Lucia Di Maio, Elena Groppali, Laura Gualtieri, Laura Martelli, Maria Luisa Melzi, Maddalena Migliavacca, Aurelio Nova, Francesco Peia, Maria Antonietta Pelagatti, Ambrogina Pirola, Ferdinando Ragazzon, Giulia Ramponi, Patrizia Rogari, Claudio Ronconi, Alessandra Sala, Martina Saruggia, Federica Zanetto. 